\section{MARINE Streptomyces SP. UKMCC_PT15 PRODUCING UNDECYLPRODIGIOSIN WITH ALGICIDAL ACTIVITY}

Zaima Azira Zainal Abidina*, Asmat Ahmadb, Jalifah Latipc, Gires Usupd

aDepartment of Biotechnology, Kulliyyah of Science, International Islamic University Malaysia, Malaysia

bSchool of Biosciences and Biotechnology, Faculty of Science and Technology, Universiti Kebangsaan Malaysia

cSchool of Chemical Sciences and Food Technology, Faculty of Science and Technology, Universiti Kebangsaan Malaysia aSchool of Environmental and Natural Resource Sciences, Faculty of Science and Technology, Universiti Kebangsaan Malaysia
Article history

Received

7 July 2015

Received in revised form

9 October 2015

Accepted

31 January 2016

*Corresponding author zzaima@iium.edu.my

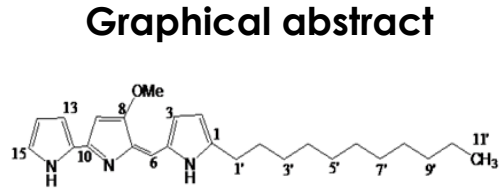

\begin{abstract}
Marine actinomycetes are now in demand as they are capable of producing unique and novel compounds with wide biological activities. Marine Streptomyces sp. UKMCC_PT15 previously isolated from seawater collected in Pulau Tinggi, Johor was used in this study. Molecular identification showed high similarity of Streptomyces sp. UKMCC_PT15 with Streptomyces fradiae and S. diastaticus subsp. ardesiacus based on partial sequence of $16 \mathrm{~S}$ rRNA gene. Further characterisations of this bacterium include spore morphology using SEM, growth on various media, salt tolerance test and carbon utilisation profile. This bacterium had straight spore chain with smooth-surfaced spores and was able to tolerate up to $11 \% \mathrm{NaCl}$ with capabilities of utilising $>40$ carbon sources. Undecylprodigiosin $\left(\mathrm{C}_{25} \mathrm{H}_{35} \mathrm{~N}_{3} \mathrm{O}\right)$ was successfully purified through succession of column chromatography and finally using HPLC. Structure elucidation was confirmed through NMR spectroscopy, MS and comparison with established data. This compound demonstrated strong antibacterial activities against S. aureus, B. subtilis and C. albicans but weak antibacterial activities against E. coli, P. aeruginosa and Methicillin-resistant $S$. aureus (MRSA), Interestingly, undecylprodigiosin also demonstrated algicidal activity when tested against toxic dinoflagellates, A. minutum and $P$. bahamense, both of which are responsible for harmful algal blooms (HABs). Undecylprodiogiosin with concentration of $10-100 \mu \mathrm{g} / \mathrm{ml}$ gave $\sim 100 \%$ algicidal activity against both dinoflagellates. Further testing with undecylprodigiosin concentration $<10 \mu \mathrm{g} / \mathrm{ml}$ showed that undecylprodigision was capable of killing significantly high numbers of both dinoflagellates, giving a high algicidal activity. Findings from this study suggested the potential use of undecylprodigiosin as algicidal agent which could be used for the mitigation of HABs.
\end{abstract}

Keywords: Marine Streptomyces, undecylprodigiosin, antibacterial , algicidal

(C) 2016 Penerbit UTM Press. All rights reserved

\subsection{INTRODUCTION}

Bacteria within the order Actinomycetales (actinomycetes) belonging to the class Actinobacteria, are Gram positive bacteria with high
$\mathrm{G}+\mathrm{C}$ content. They are filamentous with some having the ability to produce spores. Actinomycetes are common soil inhabitants and they generally have a complex life cycle, filamentous morphology, and are renowned for the production of structurally diverse 
and biologically active small organic molecules commonly referred to as secondary metabolites [1]. On the premise of finding novel and unique secondary metabolites, researchers are exploring new locations due to re-discovery of similar compounds from soil actinomycetes. New and underexplored locations such as the ocean, mangrove, desert and dunes are considered as new resources of actinomycetes.

The genus Streptomyces represents the most important genus in the production of medically and industrially valuable molecules. Approximately over 500 species belong to this genus Streptomyces, the largest number of species in nature. About $75 \%$ of antibiotics in the market currently are produced from this genus [2]. Streptomyces has the ability to produce a wide range of bioactive molecules which includes antibiotics, immunosuppressant agents, anticancer agents, antimalarial agents, enzyme inhibitors and others [3]. Owing to this unique capability of producing different bioactive molecules makes this genus remained unchallenged without a potential competitor from other microbial group.

Marine Streptomyces species are widely distributed in marine organisms such as in the fishes, molluscs, sponges, seaweeds, besides sediments and seawater as they can produce abundant spores that are readily dispersed. Furthermore, their unique capabilities of producing metabolites of commercial interest make them desirable to be pursued in search of novel metabolites despite the fact that there is still perception that marine Streptomyces will produce the same compounds as their terrestrial counterpart. Marine actinomycetes are prolific secondary metabolite producers, and majority of the compounds discovered are produced from the single genus Streptomyces. Many studies have shown that besides producing metabolites that are known from the terrestrial, marine Streptomyces are also capable to produce new metabolites [4],[5]. The past decades have witnessed the increasing number of novel metabolites possessing potent bioactivity isolated from marine-derived Streptomyces [6]. Marine Streptomyces are also capable of producing a variety of bioactive metabolites that possess a wide range of activities such as antibacterial, antifungal, antitumor, immunosuppressant agent, and many more. Examples of metabolites produced by marine Streptomyces include peptides, quininones, terpenes, macrolides, polyketides and enzyme inhibitor [7], [8].

Although many compounds have been described for actinomycetes, the algicidal potential of actinomycetes is yet to be explored. To date, few studies described the algicidal activity of the actinomycetes themselves or the compounds derived from them against microalgae particularly in mitigating harmful algal blooms (HABs) [9], [10], [1 1 ]. Harmful algal blooms (HABs) commonly known as red tides are caused by rapid growth and accumulation of microalgae in the ocean. HABs pose significant adverse impact on the marine environments, aquaculture activities and the public health.
Alexandrium minutum and Pyrodinium bahamense are among the important member of paralytic shellfish toxin (PST)-producing marine dinoflagellates especially in tropical waters. Pyrodinium bahamense produces saxitoxin and other toxin derivatives while Alexandrium minutum produces gonyautoxins (GTX), all of which cause Paralytic Shellfish Poisoning (PSP) when human ingest shellfish especially filter-feeding bivalves because they accumulate toxins as they fed this organism [12]. In the context of harnessing marine actinomycetes full potential, this study aimed at assessing the potential of compound produced by this bacterium as antimicrobial and algicidal agents beside identification and characterisation of marine Streptomyces sp. UKMCC_PT15.

\subsection{METHODOLOGY}

\subsection{Molecular Identification of and Characterisation of Marine Streptomyces UKMCC_PT15}

Marine Streptomyces UKMCC-PT15 was previously isolated from seawater collected in Pulau Tinggi, Johor. Genomic DNA Streptomyces UKMCC_PT15 was extracted using Wizard® Genomic DNA Purification Kit (Promega) following the manufacturer's protocol. PCR amplification of 16S rRNA gene was performed on the extracted DNA using the following set of primers: 27F (5'-AGAGTTTGATCCTGGCTCTCAG-3') and 1492R (5'-GGTTACCTTGTTACGACTT-3'). The PCR reaction was carried out using under the following conditions: initial denaturation at $94^{\circ} \mathrm{C}$ for $5 \mathrm{~min}$, followed by 30 cycles of $94^{\circ} \mathrm{C}$ for $30 \mathrm{~s}, 55^{\circ} \mathrm{C}$ for $60 \mathrm{~s}$ and $72^{\circ} \mathrm{C}$ for $4 \mathrm{~min}$; and extension step at $72^{\circ} \mathrm{C}$ for $10 \mathrm{~min}$. The amplification products were confirmed using $1 \%$ agarose gel and sent to $1^{\text {st }}$ Base Laboratory, Malaysia for sequencing.

Several media were used for growth and cultural characterisation of Streptomyces UKMCC_PT15 namely, SYE (Starch-Yeast Extract-Seawater), Marine Agar (MA), ISP2, ISP3, ISP4, Czapek's agar, Gause Synthetic Agar, Nutrient agar (NA) and Potato dextrose agar (PDA). All plates were incubated at $30^{\circ} \mathrm{C}$ for 2-3 weeks. The colour of aerial and substrate mycelia, the presence/absent of diffusible pigment and growth of Streptomyces UKMCC_PT15 were observed after the incubation period.

For salt tolerance test, the medium used for this purpose was SYE without the seawater but with the addition of different amount of $\mathrm{NaCl}$ in the range of $1 \%-12 \%$. The plates were incubated at $30^{\circ} \mathrm{C}$ for $2-3$ weeks.

Scanning electron microscopy (SEM) was performed on 7-day old culture grown on SYE agar to assess the morphological characteristics. Colonies on plate was cut into $1 \mathrm{~cm} \times 1 \mathrm{~cm}$ and fixed in $0.1 \%$ glutaraldehyde for overnight. Serial dehydration from $30 \%$ to $100 \%$ was performed followed by critical point drying (CPD). Before viewing, samples were sputtered with gold and viewed with SEM Philips XL 300. 
Carbon utilisation was determined using The Biolog GP2 MicroPlate following the manufacturer's instructions. The spores were collected and resuspended in a GP Inoculating Fluid and the turbidity was adjusted to $20 \%$. Thioglycollate solution was then added to the solution to prevent the cells from clumping together. About $150 \mu$ of the cells suspension was pipetted into each wells of GP2 MicroPlate. The MicroPlate was incubated at $30^{\circ} \mathrm{C}$ for 2 days. The MicroPlate was read using Biolog MicroStation ${ }^{\mathrm{TM}}$ and compared to the GP Database.

\subsection{Purification and Structure Elucidation of Undecylprodigiosin}

Streptomyces sp. UKMCC_PT15 was cultured in liquid medium of SYE and incubated at $30^{\circ} \mathrm{C}$ for 7 days with shaking at $150 \mathrm{rpm}$. The cells were harvested and extracted with methanol overnight, followed by evaporation to dryness using a rotary evaporator. Crude extract was subjected to flash column chromatography and sephadex LH-20 column chromatography and finally, undecylprodigiosin was purified using HPLC with diode array detector L-7455 (Hitachi) and a Biosilß $\mathrm{C}_{18}$ column $(10 \times 250 \mathrm{~mm}, 5 \mu)$ (Bio-Rad) with mobile phase comprised of $95 \%$ methanol and $5 \%$ of $0.1 \%$ acetic acid and flowrate of $3 \mathrm{ml} / \mathrm{min}$ at a wavelength range of $200-800 \mathrm{~nm}$. Structure elucidation of compound undecylprodigiosin was conducted through NMR spectroscopy $\left({ }^{1} \mathrm{H},{ }^{13} \mathrm{C}, \mathrm{HSQC}, \mathrm{HMBC}\right.$ and COSY), FTIR and ESI-MS.

\subsection{Evaluation of Antimicrobial Activities}

Disc diffusion assay was carried out following the method as described by Kirby-Baver et al. [13] with modification. Cell suspension of the test microorganism was prepared following a 0.5 McFarland turbidity standard. Test microorganisms used were as follows: E. coli ATCC 10536, P. aeruginosa ATCC 27853, P. mirabilis ATCC 12453, S. aureus ATCC 11632, MRSA N12, B. subtilis ATCC 11774 and C. albicans from Institute for Medical Research, Malaysia (IMR). Mueller Hinton agar was then inoculated with the test microorganism followed by placing the $6 \mathrm{~mm}$ disc containing $20 \mu \mathrm{g}$ of undecylprodigiosin as well as disc containing reference antibiotic (chloramphenicol) on the agar. The agar plate was inoculated at $37^{\circ} \mathrm{C}$ (bacteria) for 24 hours or $28^{\circ} \mathrm{C}$ (C. albicans) for 2 days. The test was carried out in duplicate. The antimicrobial activity was determined as a clear zone of inhibition surrounding the disc with the diameter expressed in $\mathrm{mm}$.

\subsection{Evaluation of Algicidal Activity}

Alexandrium tamarense and Pyrodinium bahamense cultures used in this study were from marine microalgae culture collection at Universiti Kebangsaan Malaysia. The cultures were maintained in sterile ES-DK medium prepared with natural sea water and incubated at $26^{\circ} \mathrm{C}$ under a $14 \mathrm{~h}: 10 \mathrm{~h}$ light dark cycle. Both dinoflagellates in the logarithmic growth phase was diluted with ESDK medium to a final density of $2 \times 10^{4}$ cells $/ \mathrm{ml}$. ESDK medium $(1 \mathrm{ml})$ with various concentrations of undecylprodigiosin were added to each tested algal suspension $(1 \mathrm{ml})$ in the well of 6-well plate. The final concentrations of undecylprodigiosin used were 1 to $100 \mu \mathrm{g} / \mathrm{ml}$. After incubation at $26^{\circ} \mathrm{C}$ for $24 \mathrm{~h}$, the number of viable algal cells was determined using a hemocytometer at magnification of $\times 100$. The algicidal activity was calculated using the following formula:

$$
\text { Algicidal activity }(\%)=\left(1-\frac{N_{t}}{N_{0}}\right) \times 100
$$

$\mathrm{N}_{t}=$ viable individuals of algal after the treatment $\mathrm{N}_{0}=$ initial individuals of algal

\subsection{RESULTS AND DISCUSSION}

\subsection{Marine Streptomyces sp. UKMCC_PT15}

Partial sequence of Streptomyces sp. UKMCC_PT15 was deposited to GenBank under accession number JF950277. Comparison with GenBank database showed Streptomyces sp. UKMCC_PT15 had high similarity (98\%) with S. fradiae and S. diastaticus subsp ardesiacus. SEM image revealed straight spore chain with smooth-surfaced spores (Figure 1). Streptomyces SP. UKMCC_PT15 showed good growth on SYE, MA, PDA, ISP2 and ISP4 but poor growth on ISP3, Czapek's and Gause synthetic media. Diffusible pigment was not produced in any of the medium tested. The substrate mycelium of Streptomyces sp. PT15 was red in colour when growing on SYE, MA, PDA, ISP4, Czapek's and Gause's synthetic media but lost its red pigment when growing on NA, ISP2 and ISP3 media.

Streptomyces sp. UKMCC_PT15 could tolerate up to $11 \% \mathrm{NaCl}$ demonstrating its adaption to the marine environment. This bacterium was found to show intense dark red pigmented colonies at $4 \% \mathrm{NaCl}$ that gradually decreased as the $\mathrm{NaCl}$ concentration increased. At $8 \%$ of $\mathrm{NaCl}$, a very faint red almost pale pink colony was observed. Concentration of $\mathrm{NaCl}$ higher than $8 \%$ resulted in the disappearance of the red pigment completely. This suggested the influence of $\mathrm{NaCl}$ on the pigment production in this bacterium. Interestingly, this bacterium was able to utlize $>40$ carbon sources present in the Biolog GP2 MicroPlate, amongst them were dextrin, palatinose, glycerol, gentibiose, maltose, xylose, mannitol and mannose.

\subsection{Structure Elucidation of Undecylprodigiosin}

After a succession of column chromatography, undecylprodigiosin was successfully purified through HPLC using combination of $95 \%$ methanol and $5 \%$ of $0.1 \%$ acetic acid as mobile phase at retention time of 12 min (Figure 2). Undecylprodigiosin was a deep red 
magenta solid with maximum UV absorption at $530 \mathrm{~nm}$ in methanol.

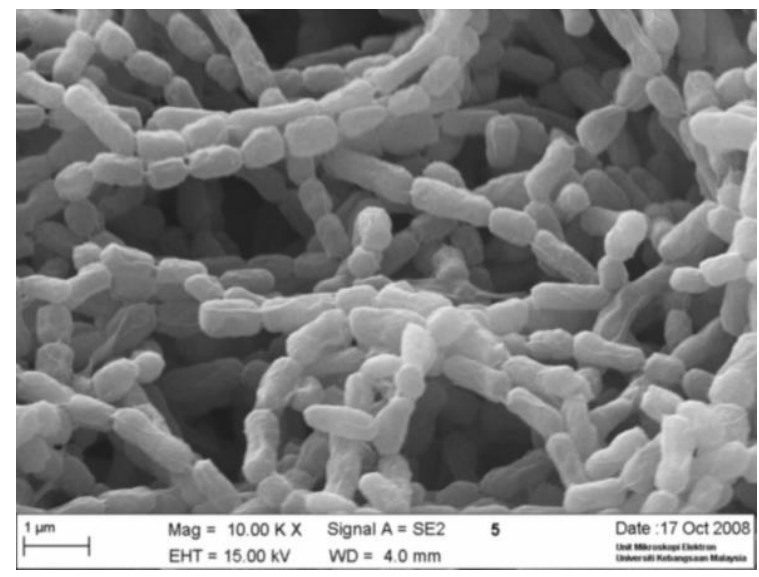

Figure 1 SEM image of Streptomyces sp. UKMCC_PT15

'H-NMR spectrum recorded aromatic protons at $\delta_{\mathrm{H}}$ $6.21,6.84,7.00,6.09$ and $6.95 \mathrm{ppm}$, a triplet at $\delta_{H} 4.01$ $\mathrm{ppm}$ corresponded to $\mathrm{OMe}$ and, $\mathrm{NH}$ protons at $\delta_{H}$ 12.85 and $\delta_{H} 12.69 \mathrm{ppm}$ (Figure 3). ${ }^{13} \mathrm{C}-\mathrm{NMR}$ spectrum recorded 13 carbons corresponded to the pyrrole rings - $\delta c 93.0,111.9,112.5,116.3,117.8,121.3,122.0$, $125.9,127.5,129.2,148.7,153.1$ and $166.2 \mathrm{ppm}$. OMe was observed at $\delta c 58.7 \mathrm{ppm}$ and there was an overlap at $\delta c 29.7$ - 31.9 ppm. Further NMR experiments - HSQC, HMBC and COSY were carried out to elucidate the structure of this compound. ESIMS was recorded at $394.28 \mathrm{~m} / \mathrm{z}\left([\mathrm{M}+\mathrm{H}]^{+}\right)$. Based on NMR spectroscopy results, ESI-MS data and by comparison of the data with the published data [14] the compound was established as undecylprodigiosin $\mathrm{C}_{25} \mathrm{H}_{35} \mathrm{~N}_{3} \mathrm{O}(393.5 \mathrm{~g} / \mathrm{mol})$.

Prodiginines are red tripyrrole antibiotics containing a pyrrolylpyrromethane skeleton. Members of this group include prodigiosin, undecylprodigiosin, cycloprodigiosin and cyclonoylprodigiosin. Undecylprodigiosin $\left(\mathrm{C}_{25} \mathrm{H}_{35} \mathrm{~N}_{3} \mathrm{O}\right)$ is a linear tripyrrole and highly nonpolar compound. Undecylprodigiosin has maximum UV absorption of at $535 \mathrm{~nm}$ in methanol, $535 \mathrm{~nm}$ in acidic methanol, and $470 \mathrm{~nm}$ in alkaline methanol [15]. Undecylprodigiosin is produced by Streptomyces longisporus ruber, Streptoverticillium rubrireticulli, Actinomadura madurae, Streptomyces coelicolor A3(2) and Saccharopolyspora sp. [16]. Undecylprodigiosin is the major component of a mixture of at least 3 prodigiosins produced by $S$. coelicolor. The production of this undecylprodigiosin usually occur in a growth phase-dependent manner, occurring in the transition and stationary phases of liquid-growth cultures and coincides with the onset of morphological development in surface-grown cultures [17]. Although Streptomyces sp. UKMCC_PT15 showed high similarity to $S$. fradiae and $S$. diastaticus subsp ardesiacus, there was no report on the production of undecylprodigiosin from these two Streptomyces species. Initially, it was thought that
Streptomyces sp. UKMCC_PT15 to be similar with S. fradiae which is also a red pigmented bacterium, however, phenotypic and genotypic characteristics showed otherwise. S. fradiae is known to produce a variety of compounds such as actinomycin Z, tylosine, frenolicin and hybrimycin but no undecylprodigiosin was reported being produced by S. fradiae. Whereas $S$. diastaticus subsp ardesiacus is a yellow/beige pigmented bacterium and was reported to produce polyene macrolide antibiotics, rimocidin and CE-108 and antifungal, oligomycins $\mathrm{A}$ and $\mathrm{C}$. Thus, this may indicate the possibility of streptomyces sp. UKMCC_PT15 to be a new species of Streptomyces.

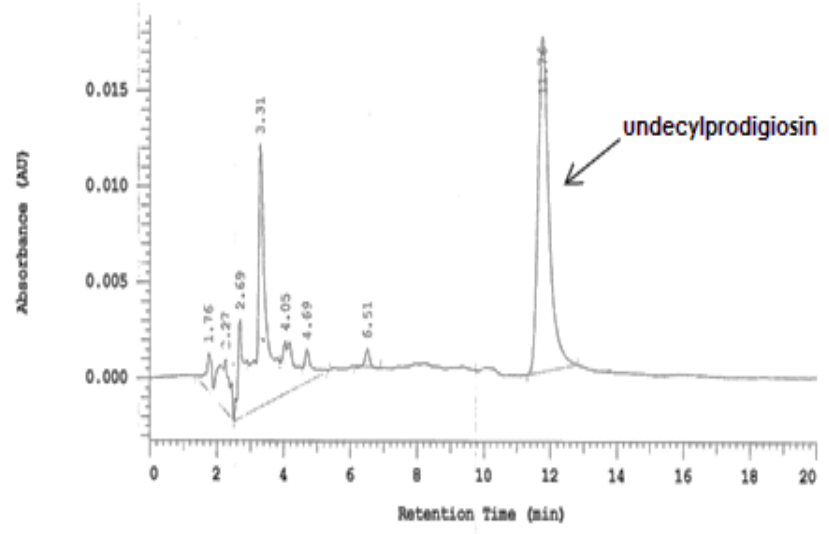

Figure 2 HPLC profile of undecylprodigiosin

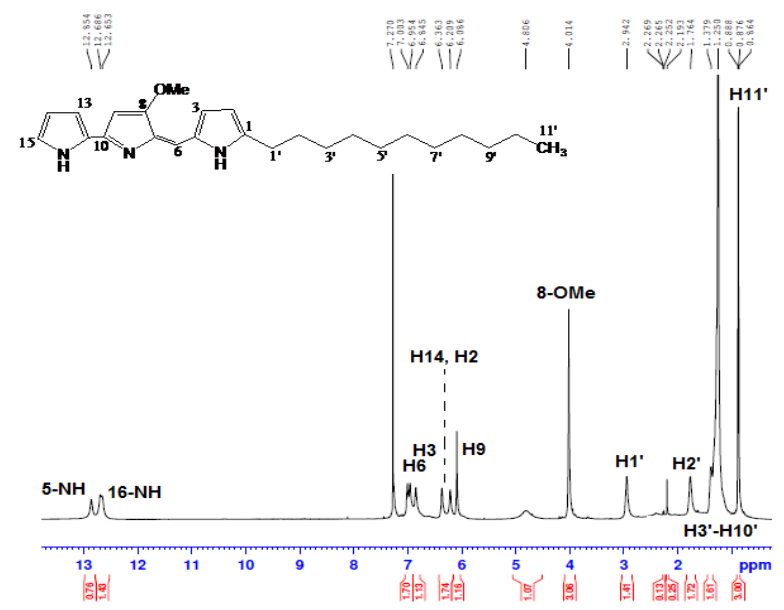

Figure 3 ' $\mathrm{H}-\mathrm{NMR}$ spectrum of undecylprodigiosin in $\mathrm{CDCl}_{3}$

\subsection{Antimicrobial Activity of Undecylprodigiosin}

Undecylprodigiosin demonstrated strong activity against S. aureus, B. subtilis and C. albicans but weak activity against E. coli, P. aeruginosa and MRSA (Table 1). Stankovic et al. [18] also reported similar findings for antimicrobial activity of undecylprodigiosin from Streptomyces sp. JS520 against B. subtilis and C. albicans. Undecylprodigiosin, however, had no effect 
on $P$. mirabilis which indicated the resistance of $P$. mirabilis to undecylprodigiosin. Difference in susceptibility between Gram-negative and gram positive bacteria towards undecyprodigiosin might be related to the cell wall morphologies. Gram-positive bacteria lacks an outer polysaccharide membrane bearing the structural lipopolysaccharide components, making the cell wall more permeable to lipophilic solutes [19]. Furthermore, the presence of porins at the outer membranes of Gram-negative bacteria which serve as membrane channels and can be closed to prevent the absorption of antimicrobial substance upon contact with low molecular weight compounds help to increase the resistance of Gramnegative bacteria [20]. Nevertheless, undecylprodigiosin holds the prospective to be developed further for antimicrobial agent.

Table 1 Antimicrobial activity of undecylprodigiosin from Streptomyces sp. PT15

\begin{tabular}{cc} 
Test microorganisms & Diameter of inhibition zone $\mathbf{( m m})$ \\
\hline E. coli & 9 \\
P.mirabilis & - \\
P. aeruginosa & 10 \\
S. aureus & 15 \\
MRSA & 10 \\
B. subtilis & 11 \\
C. albicans & 13
\end{tabular}

\subsection{Algicidal Activity of Undecylprodigiosin}

Concentrations of undecylprodigiosin in the range of 10 to $100 \mu \mathrm{g} / \mathrm{ml}$ gave almost $100 \%$ algicidal activity for A. minutum and $P$. bahamense. Undecylprodigiosin at concentrations 5 to $9 \mu \mathrm{g} / \mathrm{ml}$ gave $>95 \%$ and $>90 \%$ algicidal activity for A. minutum and P. bahamense respectively. At the lowest concentration of undecylprodigiosin $(1 \mu \mathrm{g} / \mathrm{ml})$, algicidal activity was detected at $88.3 \%$ for $A$. minutum and $80.6 \%$ for $P$. bahamense (Table 2). Treatment with undecylprodigiosin caused the algal to shrink and eventually lead to cell death. Based on the results obtained, it could be implied that undecylprodigiosin was capable of killing significantly high numbers of both dinoflagellates even at low concentrations.

Numerous activities including anticancer, immunosuppressive properties and antiprotozoal had been described for undecylprodigiosin [21],[22], [23]. However, there was no report yet on the application of undecylprodigiosin as algicidal agent. In terms of algicidal potential, another member of the prodiginines named prodigiosin is more prominent compared to undecylprodigiosin as demonstrated by several studies. Prodigiosin produced by $Y$ Proteobacterium MS-02-063 was found to be able to kill Gymnodinium impudicum, Alexandrium tamarense, Cochlodinium polykrikoides, Heterosigma akashiwo and Heterocapsa circularisquama [24], and prodigiosin from Serratia marcescens was a potent algicidal agent against Nitzschia closterium,
Skeletonema costatum and Anabena flosaquae [25]. Additionally, little is known on the effectiveness of applying bacteria or compounds derived from them in mitigating Alexandrium spp. and Pyrodinium spp. Manset et al. [26] reported the use of algicidal bacteria against Pyrodinium bahamense and Alexandrium spp. through direct and indirect interactions between the algicidal bacteria and dinoflagellates. Wang et al. [27] described algicidal protein against $A$. tamarense but so far there is no report yet on algicidal compounds derived from bacteria against Pyrodinium spp. Thus, this is the first report on the use of undecylprodigiosin from Streptomyces sp. UKMCC_PT15 as algicidal agent against $A$. minutum and $P$. bahamense. Further refinement and understanding the underlying mechanism may aid in developing undecylprodigiosin as algicidal agent in mitigating harmful algal blooms.

Table 2 Algicidal activity of undecylprodigiosin from Streptomyces sp. PT15 against $A$. minutum and $P$. bahamense

\begin{tabular}{ccc}
\hline \multirow{2}{*}{$\begin{array}{c}\text { Undecylprodigiosin } \\
(\boldsymbol{\mu g} / \mathbf{m l})\end{array}$} & \multicolumn{2}{c}{ Algicidal Activity (\%) } \\
\cline { 2 - 3 } & $\begin{array}{l}\text { Alexandrium } \\
\text { minutum }\end{array}$ & $\begin{array}{c}\text { Pyrodinium } \\
\text { bahamense }\end{array}$ \\
\hline 1 & 88.3 & 80.6 \\
2 & 90.1 & 83.2 \\
3 & 92.5 & 86.0 \\
4 & 93.0 & 88.7 \\
$5-9$ & $>95.0$ & $>90.0$ \\
$10-100$ & 99.9 & 99.8 \\
\hline
\end{tabular}

\subsection{CONCLUSION}

In conclusion, undecylprodigiosin purified from marine Streptomyces sp. UKMCC_PTI 5 holds the potential not only as antimicrobial agent but also as algicidal agent against the harmful algae $A$. minutum and $P$. bahamense. Assessment on the practical aspects may help to improve the utilisation of undecylprodigiosin in mitigating harmful algal bloom in the field.

\section{Acknowledgement}

We would like to acknowledge the Ministry of Higher Education, Malaysia for the financial support under grant UKM-GUP-KPB-08-32-138.

\section{References}

[1] Berdy, J. 2005. Bioactive Microbial Metabolites. Journal of Antibiotics. 58(1):1-26.

[2] Baltz, R. 2008. Renaissance in Antibacterial Discovery from Actinomycetes. Current Opinion in Pharmacology. 8: 1-7. 
[3] Subramani, R. and Aalsberg, W. 2012. Marine Actinomycetes: An Ongoing Source of Novel Bioactive Metabolites. Microbiological Research. 167(10):571-580.

[4] Motohashi, K., Takagi, M. and Shin-ya, K. 2010. Tetracenoquinocin and 5-Iminoaranciamycin from A Sponge-derived Streptomyces sp. Sp080513GE-26. Journal of Natural Products. 73: (4):755-758.

[5] Pérez, M., Crespo, C., Schleissner, C., Rodríguez, P., Zúñiga, P. and Reyes, F. 2009. Tartrolon D, A Cytotoxic Macrodiolide from the Marine-derived Actinomycete Streptomyces sp. MDG-04-17-069. Journal of Natural Products. 72: 2192-2194.

[6] Dharmaraj, S. 2010. Marine Streptomyces as A Novel Source of Bioactive Substances. World Journal of Microbiology and Biotechnology. 26: 2123-2139.

[7] Hughes C.C., Kauffman, C.A., Jensen, P.R. and Fenical, W. 2010. Structures, Reactivities, and Antibiotic Properties of the Marinopyrroles A-F. The Journal of Organic Chemistry. 75 (10): 3240-3250.

[8] Cho, J.Y., Kwon, H.C., Williams, P.G., Kauffman, C.A. Jensen, P.R. and Fenical, W. 2006. Actinofuranones A and B, Polyketides from A Marine-derived Bacterium Related to the Genus Streptomyces (Actinomycetales). Journal of Natural Products. 69(3): 425-428.

[9] An, X., Zahou, R., Zhang, H., Li, Y., Zheng, Yu, Z., Fu, L. and Zheng, T. 2015. Discovery of An Algicidal Compound from Brevibacterium sp. BSOl and Its Effect on A Harmful Algal Bloom-causing Species Alexandrium tamarense. Frontiers of Microbiology. doi.org/10.3389/fmicb.2015.01235

[10] Somdee, T., Sumalai, N. and Somdee, A. 2013. A novel Actinomycete Streptomyces aurantiogriseus with algicidal activity against the toxic cyanobacterium Microcystis aeruginosa. Journal of Applied Phycology. 25(5): 1587-1594.

[11] Bai, S.J., Huang, L., Su, J.Q., Tian, Y. and Zheng, T. L. 2011. Algicidal Effects of a Novel Marine Actinomycete on the Toxic Dinoflagellate Alexandrium tamarense. Current Microbiology. 62(6): 1774-1781.

[12] Lim, P.T., Usup, G. and Leaw, C.P. 2012. Harmful Algal Blooms in Malaysian Waters. Sains Malaysiana 41 (12): 15091515.

[13] Kirby-Baver, W.M.M., Sherris, J.C. and Turck, M. 1966. Antibiotic Susceptibility Testing by A Standard Single Disk Method. American Journal of Clinical Pathology 36: 493496.

[14] Liu, R., Cui, C.-B., Duan, L. Gu, Q.-Q. and Zhu, W.-M. 2005. Potent In Vitro Anticancer Activity of Metacycloprodigiosin and Undecylprodigiosin from A Sponge-derived Actinomycete Saccharopolyspora sp. nov. Archives of Pharmacal Research. 28(12): 1341-1344.

[15] Stankovic, N., Senerovic, L., llic-Tomic, T., Vasiljevic, B. and Nikodinovic-Runic, J. 2014. Properties and Applications of
Undecylprodigiosin and Other Bacterial Prodigiosins. Applied Microbiology and Biotechnology. 98:3841-3858.

[16] Williamson, N.R., Fineran, P.C., Leeper, F. and Salmond, G.P.C. 2006. The Biosynthesis and Regulation of Bacterial Prodiginines. Nature Reviews Microbiology. 4(12): 887-899.

[17] Luti, K.J.K. and Mavituna, F. 2010. Streptomyces coelicolor Increases the Production of Undecylprodigiosin When interacted with Bacillus subtilis. Biotechnology Letters. 33(1): 113-118.

[18] Stankovic, N., Radulovic, V., Petkovic, M., Vuckovic. I. Jadranin, M. Vasiljevic, B. and Nikodinovic-Runic,J. 2012. Streptomyces sp. JS520 Produces Exceptionally High Quantities of Undecylprodigiosin with Antibacterial, Antioxidative and UV-protective Properties. Applied Microbiology and Biotechnology. 96:1217-1231.

[19] Silhavy, T. J., Kahne, D. and Walker. 2010. The Bacterial Cell Envelope. Cold Spring Harbor Perspectives in Biology. 2:131.

[20] Pages, J. 2004. Role of Bacterial Porins in Antibiotic Susceptibility of Gram-negative Bacteria. In Benz, R. (ed.) Bacterial and Eukaryotic Porins: Structure, Function, Mechanisms. (1 ${ }^{\text {st }}$ ed., pp. 41-59).Weinheim: Wiley-VCH.

[21] Ho, T.F., Ma, C.J., Lu, C.H., Tsai, Y.T., Wei, Y.H., Chang, J.S Lai, J.K., Cheuh, P.J., Yeh, C.T., Tang, P.C., Chang, J., Ko J.L., Liu, F.S., Yen, H. and Chang, C.C. 2007. Undecylprodigiosin Selectively Induces Apoptosis in Human Breast Carcinoma Cells Independent of p53. Toxicology and Applied Pharmacology. 225:318-328.

[22] Williamson, N. R., Fineran, P.C., Gristwood, T., Chawral, S.R., Leeper, F.J. and Salmond, G.P.C. 2007. Anticancer and Immunosuppressive Properties of Bacterial Prodiginines. Future Medicine 2(6): 605-618.

[23] Papireddy, K., Smilkstein, M., Kelly, J.X., Salem, S.M., Alhamadsheh, M., Haynes, S.W., Challis, G.L. and Reynolds, K.A. 2011. Antimalarial Activity of Natural and Synthetic Prodiginines. Journal of Medicinal Chemistry. 54:5296-5306.

[24] Nakashima, T., Miyazaki, Y., Matsuyama, Y., Muraoka, W. Yamaguchi, K. and Oda, T. 200). Producing Mechanism of An Algicidal Compound Against Red Tide Phytoplankton in a Marine Bacterium Y-proteobacterium. Applied Microbiology and Biotechnology. 73:684-690.

[25] Liu, B.Y., Wei, D.Z., Lu, S.R, Zhou, W.Y., Shen, Y.I.X.R. and Wang, J.H . 2010. Algicidal Activity of Prodigiosin Against Harmful Algae. China Environmental Science. 30:477-482

[26] Manset, K.J.V., Azanza, R.V. and Onda, D.F.L. 2013. Algicidal Bacteria from Fish Culture Areas in Bolinao, Pangasinan, Norhern Philippines. Journal of Environmental Science and Management. Special Issue 1.

[27] Wang, B., Yang, X.R., Lu, J. Zhou, Y., Su, J., Tian, Y., Zhang, J., Wang, G. and Zheng, T. 2012. A Marine Bacterium Producing Protein with Algicidal Activity Against Alexandrium tamarense. Harmful Algae. 13: 83-88. 